\title{
Simultaneous bidirectional message transmission in a chaos-based communication scheme
}

\author{
Raúl Vicente and Claudio R. Mirasso \\ Departament de Física, Universitat de les Illes Balears, E-07122 Palma de Mallorca, Spain \\ Ingo Fischer \\ Department of Applied Physics and Photonics, Vrije Universiteit Brussel, Pleinlaan 2, B-1050 Brussel, Belgium \\ Received July 28, 2006; revised October 11, 2006; accepted October 25, 2006; \\ posted November 6, 2006 (Doc. ID 73556); published January 26, 2007 \\ We introduce a chaos-based communication scheme allowing for bidirectional exchange of information. By \\ coupling two semiconductor lasers through a partially transparent optical delay dynamics is induced in both \\ lasers. We numerically demonstrate that this dynamics can be identically synchronized, and moreover, in- \\ formation introduced on both ends of the link can be simultaneously transmitted. This scheme allows one to \\ negotiate a key through a public channel. (C) 2007 Optical Society of America \\ OCIS codes: 190.3100, 140.1540.
}

Chaotic waveforms have recently been discovered to be attractive for many applications, including communications, lidar, and coherence tomography. Their attractive features are their broadband spectrum and the possibility of synchronizing them. In optical communication systems, chaotic signals have recently been utilized to encode information, providing added security (see, e.g., Refs. 1-3 and references therein). The waveform generated by a semiconductor laser rendered chaotic, e.g., by delayed optical feedback, has been successfully used as an information carrier to encrypt small-amplitude messages onto or into it. The signal is then transmitted toward a receiver laser that is able to synchronize to the chaotic waveform. ${ }^{4}$ Very recently, a field experiment performed in a metropolitan area network of the city of Athens (Greece) confirmed the potential of this technique. ${ }^{5}$ While most of the schemes used up to now considered unidirectional transmission of information between the sender and the receiver, the development of a bidirectional communication channel is highly desirable.

Bidirectionally coupled semiconductor lasers have been found to be of considerable interest during the past few years, mostly as paradigms for delaycoupled oscillators. It has been found that two lasers exhibit chaotic emission when they are coupled with a significant delay in a face-to-face configuration., ${ }^{6,7}$ The two lasers even synchronize, however, developing a leader-laggard-type dynamics with one laser following the other by the coupling delay. ${ }^{6}$ This symmetry breaking complicates the simultaneous transmission of information in both directions.

In this Letter we propose a strikingly simple scheme that allows for simultaneous bidirectional transmission of information encoded into a chaotic carrier generated by coupled lasers, following the idea of Ref. 8. In parallel to this work, the use of two semiconductor lasers operating in a chaotic regime induced by self-optical feedback and bidirectional coupling is considered to encrypt information. ${ }^{9}$ Our scheme is depicted in Fig. 1. Two semiconductor lasers (SL1 and SL2) are mutually coupled through a partially transparent mirror (M) placed in the pathway connecting both lasers. Due to the mirror the light injected into each laser is the sum of its delayed feedback from mirror $\mathrm{M}$ and the light coming from the other laser. The coupling coefficients and the feedback strengths have been chosen such that the lasers operate in a chaotic regime. We study a situation in which both the feedback and coupling times are larger than the typical time scale of the lasers given by the relaxation oscillation period. With this configuration identical synchronization between the dynamics of both lasers can be obtained for arbitrary distances between the lasers. Moreover, we find that the position of the mirror is not relevant for the synchronization quality. Even for strongly asymmetric positioning of the mirror we still obtain identical synchronization, with temporal offset given by the difference of the corresponding delay times. The reflection and transmission characteristics of the mirror turn out not to be critical for synchronization, provided that the transmission coefficient is above a threshold value that guarantees synchronization to occur. Due to the condition $T_{i}+R_{i}=1$, where $T_{i}$ and $R_{i}$ are the transmission and reflection coefficients of the mirror, which is satisfied for the two branches of Fig. 1 , it is guaranteed that both lasers receive the same levels of light injection.

Numerical simulations of the system are performed using a set of equations that are valid for

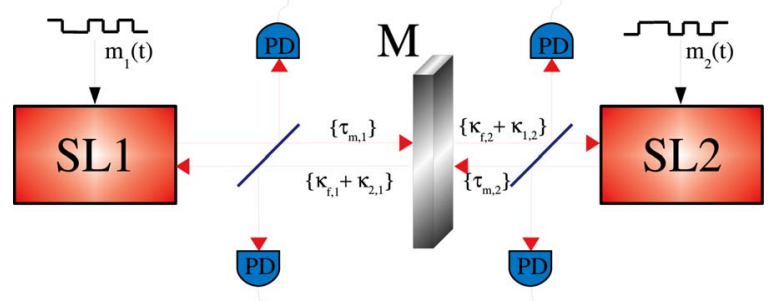

Fig. 1. (Color online) Scheme of two semiconductor lasers coupled through a partially transparent mirror: PD, photodiode; $m_{1,2}$, encoding messages. 
small to moderate values of the feedback strengths according to Ref. 10. The set of equations for SL1 reads as

$$
\begin{aligned}
\dot{E}_{1}= & i \Delta \omega_{1} E_{1}+\frac{1}{2}(1+i \alpha) G\left(N_{1},\left\|E_{1}\right\|\right) E_{1} \\
& +\kappa_{2,1} \exp \left(i \phi_{2,1}\right) E_{2}\left(t-\tau_{m, 1}-\tau_{m, 2}\right) \\
& +\kappa_{f, 1} \exp \left(i \phi_{m, 1}\right) E_{1}\left(t-2 \tau_{m, 1}\right), \\
\dot{N}_{1}= & \frac{I_{1}}{e}-\gamma_{e} N_{1}-G\left(N_{1},\left\|E_{1}\right\|^{2}\right)\left\|E_{1}\right\|^{2},
\end{aligned}
$$

while a similar modeling stands for SL2 when indices 1 and 2 are exchanged. $E_{i}(t)$ is the complex amplitude of the optical field generated by laser $i$, and $N_{i}$ represents the corresponding carrier's number. $\left\|E_{i}\right\|^{2}$ denotes the number of photons inside the cavity. The gain function, $G\left(N_{i},\left\|E_{i}\right\|^{2}\right)$, is given by

$$
G\left(N_{i},\left\|E_{i}\right\|^{2}\right)=\frac{g\left(N_{i}-N_{0}\right)}{1+s\left\|E_{i}\right\|^{2}}-\gamma .
$$

The internal laser parameters are assumed to be identical for the two lasers: the linewidth enhancement factor $\alpha=3$, the differential gain $g=1.2$ $\times 10^{-5} \mathrm{~ns}^{-1}$, the transparency inversion $N_{0}=1.25$ $\times 10^{8}$, the saturation coefficient $s=5 \times 10^{-7}$, the photon decay rate $\gamma=496 \mathrm{~ns}^{-1}$, and the carrier decay rate $\gamma_{e}=0.651 \mathrm{~ns}^{-1}$. In the following the bias current has been fixed to $2.2 I_{\text {th }}\left(I_{\text {thr }}=17.3 \mathrm{~mA}\right)$ in both lasers. At this current level the relaxation oscillation frequency is $\sim 6 \mathrm{GHz}$. The frequency detuning $\Delta \omega_{i}=0$. The coupling and feedback strengths are chosen to be symmetric, corresponding to a $50 \%$ transparent mirror; $\kappa_{1,2}=\kappa_{2,1}=\kappa_{f, 1}=\kappa_{f, 2}=20 \mathrm{~ns}^{-1}, \quad \sim 5 \%$ of the light is coupled and fed back, respectively. Regarding the coupling delay times, we take $\tau_{m, 1}=1.4 \mathrm{~ns}$ and $\tau_{m, 2}$ $=2.4 \mathrm{~ns}$. The phases are set to $\phi_{1,2}=\phi_{2,1}=\phi_{m, 1}=\phi_{m, 2}$ $=0 \mathrm{rad}$. The coupling phase is not important individually, only in relation to the feedback phases; if the coupling phase does not equal the semi-sum of the feedback phases, the correlation between the lasers degrades.

In the absence of any external perturbation both lasers operate in a chaotic regime, as shown in Fig. 2. From the time traces plotted in the main panel it can be clearly seen that the lasers operate in the coherence collapse regime. To be as general as possible we have placed the mirror closer to one of the lasers (SL2) to induce an asymmetry in the two branches of Fig. 1. In the inset of Fig. 2 we plot the crosscorrelation function between the output powers $P_{1}$ and $P_{2}$. It can be seen that the cross-correlation function exhibits the maximum peak at a time lag that amounts to the difference between the coupling times of both lasers with the mirror $\Delta t=\tau_{m, 2}-\tau_{m, 1}=1 \mathrm{~ns}$, with a correlation coefficient of 1 , which indicates an identical synchronization between the dynamics. When the mirror is moved to the center, the maximum of the cross-correlation function moves toward zero. ${ }^{8}$ It is worth mentioning that under small mis-

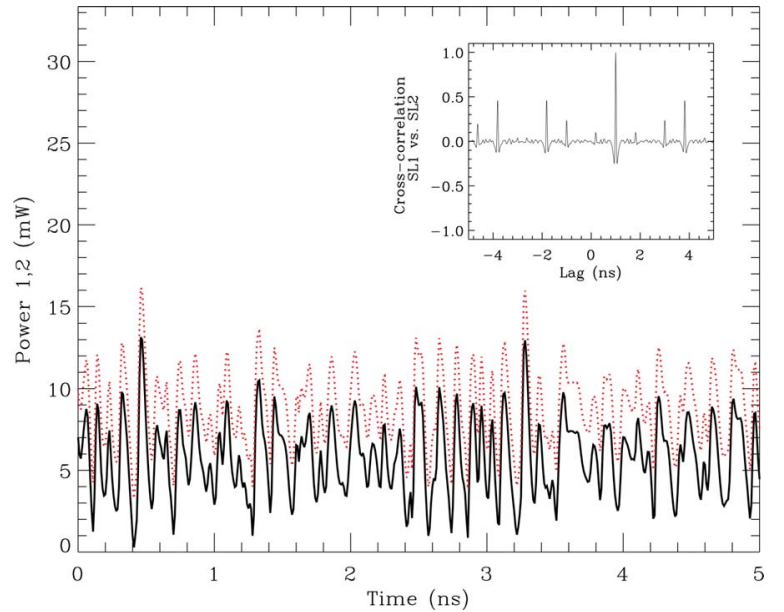

Fig. 2. Temporal traces of the optical power of SL1 (black) and SL2 (gray, red online) once the proper lag has been compensated for. The trace of SL2 has also been shifted vertically to distinguish it from that of SL1. The inset shows the cross-correlation function between the two laser outputs.
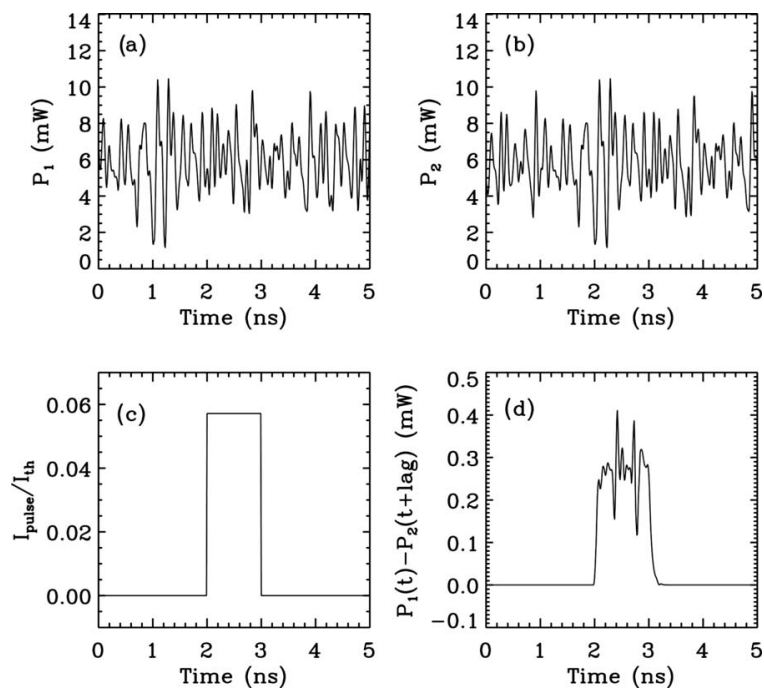

Fig. 3. (a), (b) Temporal traces of SL1 and SL2, respectively, under the injection of a current pulse shown in (c). (d) Evolution of the synchronization error.

matches between laser parameters (of a few percent) synchronization is maintained, although the correlation coefficient slightly degrades.

Once identical synchronization, as opposed to leader-laggard dynamics, has been proved, the robustness of the synchronized solution against an external perturbation is studied. To this purpose we inject a square pulse through the bias current of one of the lasers and follow the evolution of the synchronization error $P_{1}(t)-P_{2}(t+\Delta t)$. In Figs. 3(a) and 3(b) the temporal traces of the lasers are shown. The current pulse, which is injected at $t=2 \mathrm{~ns}$, has an amplitude of $0.058 I_{\text {th }}$. It is found to deteriorate the perfect correlation only while acting on SL1. As soon as the pulse propagates toward the communication channel, the mirror bidirectionally redistributes the perturbation and the synchronization solution is perfectly maintained. Even under larger perturbations (pulses with an amplitude higher than the bias current) we 
have observed that synchronization is lost only during the application of the pulse to the laser, regardless of the position of the mirror in the pathway.

Next we use the proposed scheme to simultaneously exchange information between SL1 and SL2 by using a single communication channel. We encode the information by simultaneously modulating the bias currents of both lasers with two independent pseudorandom digital messages of amplitude $0.12 I_{\text {th }}$ at $1 \mathrm{Gbit} / \mathrm{s}$. The two transmitted messages $\left(m_{1}\right.$ and $\left.m_{2}\right)$ are shown in Figs. 4(a) and 4(b), respectively. Since the amplitude of the messages is kept small, the information is well hidden within the chaotic carriers. The procedure to decipher the messages starts by subtracting the optical power of of both lasers. The synchronization error between the lasers' powers $\left[P_{1}(t)\right.$ and $\left.P_{2}(t+\Delta t)\right]$ allows one to recover the difference between the messages that have been sent [Fig. 4(d)], which reproduces the difference between the original messages [Fig. 4(c)] after the appropriate lag has been compensated for $\left[m_{1}(t)-m_{2}(t+\Delta t)\right]$ by, e.g., a variable $\mathrm{RF}$ delay line. After digitalizing this difference, only the sender of $m_{2}$ can completely recover the content of $m_{1}$ and vice versa. It is worth mentioning that the maximum encoding rate depends on the inverse of the resynchronization time after a bit arrives at one of the lasers. Under these conditions, it turns out to be $\sim 0.3 \mathrm{~ns}$. Consequently, a maximum bit rate of $\sim 3 \mathrm{Gbits} / \mathrm{s}$ could be achieved.

At this point it is worth discussing the security aspects of our scheme. Since both output powers $\left(P_{1}\right.$ and $P_{2}$ ) are accessible from the same communication channel (a simple beam splitter easily allows for separating the signals coming from SL1 and SL2), an eavesdropper could easily monitor the difference $P_{1}$ $-P_{2}$, and consequently the difference of the messages
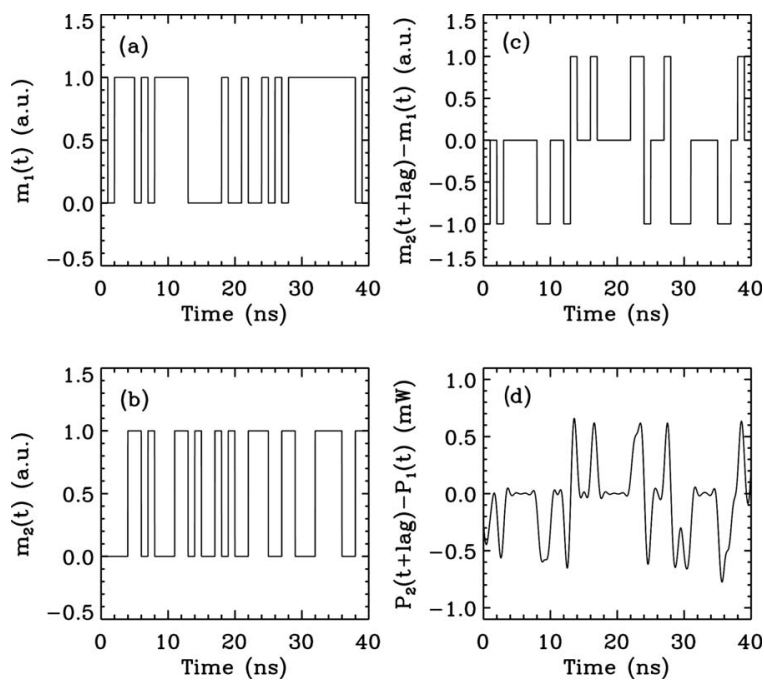

Fig. 4. Illustration of the message decryption process. (a) and (b) Original messages encoded by SL1 and SL2, respectively. (c) Subtraction of messages $\left[m_{1}(t)-m_{2}(t+\Delta t)\right]$ with a given time lag, which is reconstructed by the synchronization error $\left[P_{1}(t)-P_{2}(t+\Delta t)\right]$. This difference, (d), has been filtered with a fifth-order Butterworth filter with a cutoff frequency of $0.8 \mathrm{GHz}$. being transmitted. Thus, a level of 1 in the message difference would clearly indicate that at the proper time the bit associated with SL1 was a "1" while the one sent by SL2 was a "0." A similar argument holds when the message difference is -1 . Only when the message difference is zero (i.e., both lasers are coding the same bit), the eavesdropper has no clue as to which are the bits that are being sent. Based on this result, this type of mutually synchronized configuration could be used to simultaneously negotiate a key. ${ }^{11,12}$ Both sides of the link can agree to discard those bits that are different from each other while accepting that the key that is formed by the first $N$ bits that coincide with each other. In this way a key is encrypted with the same level of security as in a unidirectional chaos communication scheme. The main advantage of this approach resides in the fact that both sender and receiver now can negotiate a key through a public channel.

In conclusion, we have proposed a strikingly simple scheme that allows for bidirectional and simultaneous transmission of information encoded within chaotic carriers. By coupling two semiconductor lasers bidirectionally through a partially transparent mirror, we obtain identical synchronization that has been proved to be very robust. The scheme can be used to exchange an encrypted key through a public channel.

This work has been funded by the Spanish MCyT and Feder projects TEC2005-07799-C02-01 and FIS2004-00953, EC Project PICASSO IST-200534551, and the Interuniversity Attraction Pole program (IAP V/18). C. Mirasso's e-mail address is claudio@galiota.uib.es.

\section{References}

1. S. Donati and C. R. Mirasso, eds., "Feature section on optical chaos and applications to cryptography," IEEE J. Quantum Electron. 38, 1138-1205 (2002).

2. L. Larger and J. P. Goedgebuer, eds., "Cryptography using optical chaos," C. R. Phys. 5, 609-681 (2004).

3. A. Uchida, F. Rogister, J. Garcia-Ojalvo, and R. Roy, Prog. Opt. 48, 203 (2005).

4. I. Fischer, Y. Liu, and P. Davis, Phys. Rev. A 62, 011801R (2000).

5. A. Argyris, D. Syvridis, L. Larger, V. Annovazzi-Lodi, P. Colet, I. Fischer, J. Garcia-Ojalvo, C. R. Mirasso, L. Pesquera, and K. A. Shore, Nature 238, 343 (2005).

6. T. Heil, I. Fischer, W. Elsäßer, J. Mulet, and C. R. Mirasso, Phys. Rev. Lett. 86, 795 (2001).

7. H. Fujino and J. Ohtsubo, Opt. Rev. 8, 351 (2001).

8. I. Fischer, R. Vicente, J. M. Buldú, M. Peil, C. R. Mirasso, M. C. Torrent, and J. García-Ojalvo, Phys. Rev. Lett. 97, 123902 (2006).

9. E. Klein, N. Gross, N. Rosenbluh, L. Khaykovich, W. Kinsel, and I. Kanter, Phys. Rev. E 74, 046201 (2006).

10. J. Mulet, C. Masoller, and C. R. Mirasso, Phys. Rev. A 65, 063815 (2002).

11. C. H. Bennett, Phys. Rev. Lett. 68, 3121 (1992).

12. R. Mislovaty, E. Klein, I. Kanter, and W. Kinzel, Phys. Rev. Lett. 91, 118701 (2003). 